\section{Impact of ageing on problem size and proactive interference in arithmetic facts solving}

Quarterly Journal of Experimental Psychology I-II

(c) Experimental Psychology Society 2018 Reprints and permissions: sagepub.co.uk/journalsPermissions.nav DOI: $10.1177 / \mid 747021818759262$ qjep.sagepub.com

\author{
Kim Archambeau', Alice De Visscher ${ }^{2}$, Marie-Pascale Noël ${ }^{2}$ \\ and Wim Gevers'
}

\begin{abstract}
Arithmetic facts (AFs) are required when solving problems such as " $3 \times 4$ " and refer to calculations for which the correct answer is retrieved from memory. Currently, two important effects that modulate the performance in AFs have been highlighted: the problem size effect and the proactive interference effect. The aim of this study is to investigate possible age-related changes of the problem size effect and the proactive interference effect in AF solving. To this end, the performance of young and older adults was compared in a multiplication production task. Furthermore, an independent measure of proactive interference was assessed to further define the architecture underlying this effect in multiplication solving. The results indicate that both young and older adults were sensitive to the effects of interference and of the problem size. That is, both interference and problem size affected performance negatively: the time needed to solve a multiplication problem increases as the level of interference and the size of the problem increase. Regarding the effect of ageing, the problem size effect remains constant with age, indicating a preserved AF network in older adults. Interestingly, sensitivity to proactive interference in multiplication solving was less pronounced in older than in younger adults suggesting that part of the proactive interference has been overcome with age.
\end{abstract}

\title{
Keywords
}

Proactive interference; problem size; ageing; arithmetic facts; numerical cognition

Received: 26 May 2017; revised: II December 2017; accepted: 21 December 2017

\section{Introduction}

Arithmetic facts (AFs) refer to calculations for which the correct answer is retrieved from memory rather than calculated. Numerical problems (operands and answers) of AFs, such as " $3 \times 4$ ", are assumed to be stored in interconnected associative networks in long-term memory (e.g., Ashcraft, 1992; Campbell, 1995; Verguts \& Fias, 2005). Importantly, they are required for many everyday life situations, for instance, to make a recipe or to pay at the store, and are considered as the basis of more complex calculations. Given the high incidence of arithmetic difficulties throughout development, the acquisition of AFs received much attention (e.g., Geary, 1993; Jordan \& Montani, 1997). However, although fundamental, only a handful of studies (e.g., Geary \& Wiley, 1991) investigated the impact of normal ageing on AF solving.

A robust observation regarding AFs is the modulation of the performance by the size of the problem when solving AFs, i.e., the problem size effect (e.g., Campbell, 1995; De, Brauwer, Verguts, \& Fias, 2006; Stazyk, Ashcraft, \& Hamann, 1982; for a review, see Zbrodoff \& Logan, 2005). The problem size effect indicates that small problems (e.g., $2 \times 3$ ) are solved faster and with fewer errors than large problems $(7 \times 8)$. This effect is assumed to reflect a memory retrieval component of processing

\footnotetext{
'Center for Research in Cognition \& Neurosciences (CRCN), Université Libre de Bruxelles (ULB), Brussels, Belgium 2Psychological Sciences Research Institute, Université cathtolique de Louvain, Louvain-la-Neuve, Belgium

Corresponding author:

Kim Archambeau, Center for Research in Cognition \& Neurosciences $(\mathrm{CRCN})$, Université Libre de Bruxelles (ULB), Avenue Franklin Roosevelt 50, 1050 Brussels, Belgium.

Email: karchamb@ulb.ac.be
} 
(Allen et al., 2005; Campbell \& Graham, 1985; Campbell \& Xue, 2001; LeFevre et al., 1996; Miller, Perlmutter, \& Keating, 1984). A typical explanation of the problem size effect is that the strength of associations between operands and answers differs between AFs in the network (Ashcraft \& Guillaume, 2009; see also Allen et al., 2005): small problems present stronger associations in long-term memory and are therefore solved faster than large problems. Some authors suggested that the variation in the strength of these associations depends on the frequency of occurrence and usage of each numerical problem (Ashcraft, 1982, 1987; Ashcraft \& Christy, 1995; Hammann \& Ashcraft, 1986; McCloskey \& Lindemann, 1992). For example, Ashcraft and Christy (1995) reported that large problems are underrepresented in elementary school books compared with small problems.

Another explanation of the problem size effect was proposed in terms of differences in strategy selection between small and large problems (Campbell \& Xue, 2001; LeFevre et al., 1996; Penner-Wilger, Leth-Steensen, \& LeFevre, 2002; Imbo \& Vandierendonck, 2008; for a review, see Zbrodoff \& Logan, 2005). More specifically, small problems would be more often solved using retrieval strategies than large problems. In other words, the use of procedural strategies, which are considered as less efficient, would be more frequently used for large than for small problems.

Regarding the effects of ageing, previous studies on AF solving mainly reported similar problem size effects between young and older adults (e.g., Allen et al., 2005; Allen, Smith, Jerge, Vires-Collins, 1997; Geary, Frensch, \& Wiley, 1993; Geary \& Wiley, 1991). For instance, using multiplication production and verification tasks, Allen et al. (1997) demonstrated that only peripheral processes (i.e., encoding and response execution) varied across ages. Older adults were slower in encoding problems and executing responses than young adults. In contrast to peripheral processes, age-related invariance is observed for the problem size effect. A similar pattern of results was found with addition operations (Geary \& Wiley, 1991), as well as with subtraction operations (Geary et al., 1993). Based on these findings, it was argued that age-related differences mainly concerned peripheral processes but not fact retrieval per se, suggesting an intact AF network in older adults.

A recent study (De Visscher \& Noël, 2014) highlighted another important factor influencing the performance in AFs: the proactive interference parameter. This parameter is believed to measure the proactive interference that is progressively set throughout the learning of multiplication tables. According to these authors, the previously learned problems disturb the learning of new problems (i.e., proactive interference). This proactive interference would occur because of the physical similarities between the old and the new problems. These physical similarities come from the analogous associations of digits (i.e., the combination of the digits 0-9) composing all AFs. Problems that have more similar associations of digits with the previously learned problems would be more difficult to memorise.

Concretely, the proactive interference parameter was computed by measuring the number of similar associations between a given problem and previously learned problems. For example, when learning the multiplication $3 \times 9$ $=27$, the different combinations of two digits for this problem are 2-7, 2-3, 3-9, 3-7, 9-2, and 9-7. According to the learning order from Tables 2 to 9 , the combination of digits 2-7 has already been encountered in two previously learned problems $(2 \times 7=14$ and $3 \times 7=21)$, and therefore, 2 points are added to the proactive interference score of that problem. The combination of the digits 3-2 has been found in four previously learned problems $(2 \times 3=6,4 \times 3=12$, $3 \times 7=21$, and $3 \times 8=24$ ), and hence, 4 points are added to the proactive interference score. Similarly, the combinations of digits 3-9 $(3 \times 3=9), 3-7(3 \times 7=21)$, and 9-2 (2 $\times 9=18$ ) have been found in one previously learned problem and respectively add 1 point to the proactive interference score. Finally, the combination of the digits 7-9 has not been found in any of the previously learned problems, and so, no point is added. In sum, the total score of proactive interference for the multiplication $3 \times 9=27$ is 9 (for further details, see De Visscher \& Noël, 2014). In sum, the proactive interference parameter provides a quantitative measure of the proactive interference weight for each multiplication by measuring the overlap of digits between that problem and the previously learned ones.

The authors' results showed that beyond the size effect, the effect of interference substantially determined the performance across multiplication problems, meaning that the time to solve a multiplication increases as the level of proactive interference increases. This effect of interference has been observed in children as well as in young adults (De Visscher \& Noël, 2014; see also De Visscher, Berens, Keidel, Noël, \& Bird, 2015).

To our knowledge, no study has currently investigated the evolution of proactive interference with age in $\mathrm{AF}$ solving. In the non-numerical domain, it has repeatedly been observed that older adults have more difficulty resolving proactive interference in memory tasks (e.g., Chiappe, Siegel, \& Hasher, 2000; Jonides et al., 2000; Loosli, Rahm, Unterrainer, Weiller, \& Kaller, 2014; May, Hasher, \& Kane, 1999; Thompson-Schill et al., 2002). For instance, Jonides et al. (2000) compared age-related changes in proactive interference via the recent probes task (Monsell, 1978). In this task, participants have to memorise a set of items (target set of trial $n$; e.g., "a b c d"). Subsequently, a probe is presented, and participants have to decide whether it was one of the memorised items of that trial. This probe can either require a positive response (e.g., "a") because it was a member of the target set (positive probes) or require a negative response (e.g., "e") because it was not a member of the target set (negative 
probes). Some negative probes (e.g., "f") were presented at the previous target set (trial $n-1$; e.g., "f $\mathrm{g} \mathrm{h}$ i") producing proactive interference (they are called, recent negative probes), while the other negative probes (e.g., "k") were not recently presented. Jonides et al. (2000) demonstrated that the difference in performance between the recent negative probes and the non-recent negative probes was larger for older adults, suggesting a higher sensitivity to proactive interference with age.

The main aim of the current study was to examine the impact of normal ageing on AF solving by investigating both the problem size effect and the proactive interference effect. Related to this purpose, we explored whether the above-mentioned proactive interference parameter was still playing a role in older adults and whether AFs were affected by the increase of sensitivity to interference with ageing. To that aim, we used an independent measure of sensitivity to proactive interference to further define the architecture underlying the proactive parameter in multiplication solving. We compared the performance of participants from two different age groups (young and older adults) in a multiplication production task and in a recent probes task. We hypothesised that both the problem size effect and the proactive interference effect would determine the performance in AF solving in both groups. Considering previous studies (Allen et al., 2005; Allen et al., 1997; Geary et al., 1993; Geary \& Wiley, 1991), we expected a similar problem size effect between older and young adults. Concerning the proactive interference effect, De Visscher and Noël (2014) argued that proactive interference is taking place during the developmental stage when children are learning the AFs. Based on this argument, the amount of proactive interference can be expected to be the same or even smaller in older compared with younger adults as the time interval between the creation of the AF network and the moment of testing is by definition longer in older compared with younger adults because the AF network of older adults is built since a long time ago and may have surmounted a part of this proactive interference effect. Alternatively, if the amount of proactive interference relates to overall sensitivity to interference (e.g., Jonides et al., 2000) rather than to the passage of time since the learning of the AFs, one can expect stronger proactive interference effects in older compared with younger adults.

\section{Method}

\section{Participants}

A total of 48 participants took part in this study: 24 young adults between the age of 20 and 35 years, and 24 healthy older adults between the age of 55 and 75 years. Each volunteer was paid $€ 8$ for his or her participation. The study protocol was approved by the local ethical committee. All participants were naïve with respect to the purpose of the experiment. Participants with dementia, alcoholism, low level of education (at least primary education), history of stroke, head trauma, metabolic disorder, psychiatric disorder, and general anaesthesia in the past 6 months were excluded. In the older adults, the Montreal Cognitive Assessment (MoCA; Nasreddine et al., 2005) was used to assess global cognitive functioning to exclude subjects with potential risk of dementia (MoCA score >26). Moreover, the Geriatric Depression Scale (GDS) was used to discard subjects with high depressive symptoms (GDS score $>10$ ). All participants were French-speaking and had normal or corrected to normal vision.

Following the data acquisition and before the data analyses, four older adults were excluded because they did not meet the selection criteria presented above: two had a suspected cognitive impairment and two had suspected depressive symptoms. The final sample thus included 44 participants: 24 young adults (mean $[M]=24.5$ years, standard error $[S E]=0.75-16$ women [1 left-handed] and 8 men [ 2 left-handed]) and 20 older adults $(M=62.9$ years, $S E=1.26-12$ women [1 left-handed] and 8 men [1 lefthanded]). Young adults had more years of education than older adults, $t(41)=3.04, p<.01$. The average level of education was 16 years ( $S E=0.43$ years) and 13.70 years ( $S E=0.62$ years) in young and older adults, respectively. We therefore explored the relationship between the level of education and our variables of interest and no correlation was found (all $r<|.15|$, all $p>.350$ ).

\section{Material and procedure}

All the participants were submitted to a pool of tasks. Here, we report only the data for the multiplication production and recent probes tasks that are of interest to this study. Young and older adults were tested in a quiet room at the university or at home. All tasks were run on a 17-inch laptop computer, using Prime 2 Professional Software (Psychology Software Tools).

\section{Multiplication production task}

Participants were asked to solve the 36 multiplication problems from $2 \times 2$ to $9 \times 9$ (without the commutative pairs), including the 8 ties problems (e.g., $6 \times 6$ ). Following the method of De Visscher and Noël (2014), 14 of the nontie problems started with the smaller operand and 14 started with the larger operand, with a similar problem size (magnitude of the products) between both subsets. Multiplication problems were presented following a pseudo-random order, such that two successive problems never had the same answer or operand and that no more than three successive problems were of the same type (smaller operand first, larger operand first, or tie problem). Each trial began with a fixation point $(500 \mathrm{~ms})$ followed by 


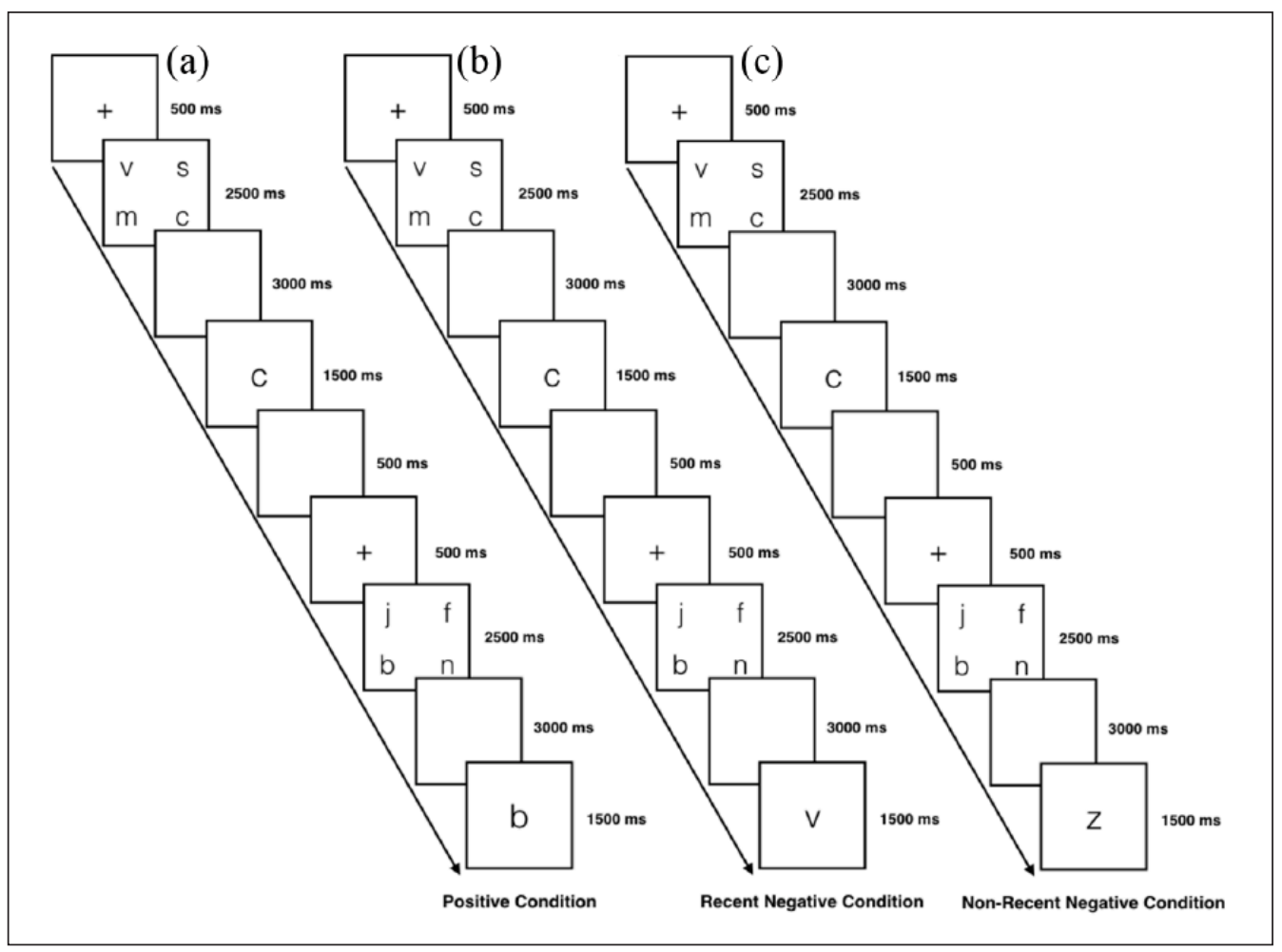

Figure I. Illustration of the design for the recent probes task with an example for (a) the positive condition, (b) the recent negative condition, and (c) the non-recent negative condition.

a multiplication problem displayed in Arabic format in black on a white background until a response was produced orally. The oral response removed the multiplication from the screen, and the next trial started after a delay of $500 \mathrm{~ms}$. A millisecond-accurate voice key was used to record vocal response latencies. Accuracy was recorded by the experimenter. Participants were instructed to answer as quickly and as accurately as possible.

\section{Recent probes task}

In the recent probes task, participants had to judge whether a probe was part of the target set previously presented. A trial started with a fixation point $(500 \mathrm{~ms})$ followed by the target set. The target set consisted of four lowercase letters (Courier New font, size 50) arranged as a square (see Figure 1). The target set remained on the screen for $2500 \mathrm{~ms}$ followed by a delay of $3,000 \mathrm{~ms}$. After this delay, a single letter probe was presented in the middle of the screen (Courier New font, size 50). Participants had to indicate, by pressing a left-sided or a right-sided button on a response box, whether the letter belonged to the current target set or not. Participants were instructed to answer as fast and as accurately as possible. The maximum response time allowed was $1500 \mathrm{~ms}$. After this delay or after a response, the next trial was initiated, with an inter-trial interval of $500 \mathrm{~ms}$. On half of the trials, the probe required a positive response because it matched a member of the target set (positive trials; see Figure 1a). On the other half, the probe required a negative response because it did not match any member of the target set (negative trials). Two types of negative trials were contrasted: in the recent negative trials, the probe did not match a target of the current target set but was a member of the previous target set (see Figure 1b) and in the non-recent negative trials, the probe did not match any targets from the current trial and was not a member of either of the previous two target sets (see Figure 1c). In total, the task included 80 trials consisting of 40 positive trials, 20 non-recent negative trials, and 20 recent negative trials. One half of the participants had to press the left button for positive trials and the right button for negative trials, whereas for the other half this response mapping was reversed. The trials were pseudo-randomly presented with no more than three consecutive identical conditions. The letters were randomly chosen among the consonants with the restriction that the letters were not perceptually similar. According to a visual similarity matrix computed by Boles and Clifford (1989), seven associations of letters (i.e., "db", "dp", “dq", “qp", "xk", "qg", and "nm") were excluded between the negative probe and the letters in the target set as well as between the letters within the target set. Finally, the arrangement of positive probes and recent negative probes was balanced between the four spatial positions of the target set. 


\section{Data analyses}

In the multiplication production task, the proactive interference parameter and the product were used as measures of the proactive interference and the size of the problems, respectively. Data were analysed using both repeatedmeasures analyses of variance (ANOVAs) and linear regressions. For the ANOVAs, a dichotomous assignment was used for each effect (low vs high interference and small vs large problem size). For the interference effect, the 36 multiplication problems were ordered according to the proactive interference parameter and the 18 least interfering were defined as "low interference" (proactive interference parameter of 7 or smaller), while the 18 more interfering were defined as "high interference" (proactive interference parameter from 8 to 25). For the problem size effect, the 18 multiplication problems with the smallest products were classified as "small" problems (products that were 25 or smaller), and the 18 multiplication problems with the largest products were classified as "large" problems (products from 27 to 81 ). In accordance with this assignment, a $2 \times 2$ factorial design was produced with 12 multiplications in small/low interfering problems as well as in large/high interfering problems conditions and with 6 multiplications in large/low interfering problems as well as in small/high interfering problems conditions (see supplementary material for further details). Therefore, we used a repeated-measures ANOVA with problem size (2: small problems, large problems) and proactive interference (2: low proactive interference, high proactive interference) as within-subjects factor and group (2: young adults, older adults) as between-subjects factor. The ANOVAs were performed on mean accuracy and the median of reaction times (RTs) of correct trials only.
For the linear regression analyses, the interference level and the problem size were coded continuously according to the proactive interference parameter and the product, respectively. First, we ran a linear regression for each group with the median of RTs as dependent variable and the problem size and proactive interference parameter as independent variables. This analysis was used to investigate which predictors determine the difficulty across multiplications. Second, we performed separate regression analyses for each participant with RTs as dependent variable and problem size, and the proactive interference parameter as predictors. The individual slopes for each independent variable reflect a measure of each individual's sensitivity to proactive interference and problem size, whereas the individual intercepts reflect an individual measure of peripheral processes (Cerella, 1991). These measures were therefore used to examine age differences in sensitivity to proactive interference, problem size, and peripheral processes.

In the recent probes task, we used a repeated-measures ANOVA with condition (3: positive, non-recent negative, and recent negative) as within-subjects factor and group (2: young adults, older adults) as between-subjects factor. This analysis was performed on the mean accuracy and the median of RTs of correct trials only. To assess the proactive interference effect, the critical comparison was the difference in performance between the non-recent negative and recent negative condition. To obtain an individual measure of proactive interference that is not confounded with general processing speed, the sensitivity to proactive interference for each participant was computed as follows ${ }^{1}$ :

$$
\text { Sensivity to interference }=\frac{\text { Median } R T \text { on recent negative trials minus Median } R T \text { on non }- \text { recent negative trials }}{\text { Median } R T \text { on non }- \text { recent negativetrials }}
$$

This measure was used to directly compare the sensitivity to proactive interference between young and older adults. Finally, a Bayesian analysis (Eidswick, 2012) using JASP software (Love et al., 2015) was conducted to confirm the null hypothesis in our effects of interest (for a complete interpretation of Bayes factors [BFs], see Wagenmakers, Wetzels, Borsboom, \& van der Maas, 2011).

\section{Results}

\section{Multiplication production task}

A repeated-measures ANOVA with problem size and proactive interference as within-subjects factor and group as between-subjects factor was run separately on accuracy (percentage of errors) and median RTs of correct trials (for an overview of all results, see Table 1). In young adults, the average error rate was $11.29 \%(S E=1.69 \%)$ and the mean of median RTs was $1,232 \mathrm{~ms}(S E=38.43$ $\mathrm{ms})$. In older adults, the mean number of errors was $7.218 \%(S E=1.85 \%)$ and the mean of median RTs was $1,037 \mathrm{~ms}(S E=42.09 \mathrm{~ms})$.

Regarding the error rate, the repeated-measures ANOVA showed a main effect of problem size, $F(1,42)=62.01, p<$ $.001, \eta_{\mathrm{p}}^{2}=.60$, indicating that participants were more accurate for small problems (error rate $=2.52 \%, S E=0.65$ ) than for large problems (error rate $=15.95 \%, S E=2.04 \%$ ). A main effect of proactive interference was also observed, $F(1,42)=32.10, p<.001, \eta_{\mathrm{p}}^{2}=.43$. Low interfering problems were better performed than high interfering problems (low interfering problems: error rate $=4.67 \%$, $S E=0.91 \%$; high interfering problems: error rate $=$ $13.80 \%, S E=1.90 \%)$. The interaction between proactive interference and problem size was significant, $F(1,42)=$ 
Table I. Descriptive (average error rate in percentage and mean of median RTs in milliseconds) and inferential statistics for the multiplication production task.

\begin{tabular}{|c|c|c|c|c|}
\hline Variables & $\begin{array}{l}\text { Error rate } \\
(\%)\end{array}$ & $\begin{array}{l}\text { Reaction } \\
\text { times (ms) }\end{array}$ & ANOVA (error rate) & ANOVA (reaction times) \\
\hline \multicolumn{5}{|l|}{ Problem size } \\
\hline Small & 2.52 & 1,003 & \multirow{2}{*}{$F(I, 42)=62.01, p<.00 I, \eta_{p}^{2}=.60$} & \multirow{2}{*}{$F(I, 42)=89.72, p<.00 I, \eta_{p}^{2}=.68$} \\
\hline Large & 15.95 & 1,266 & & \\
\hline \multicolumn{5}{|l|}{ Proactive interference } \\
\hline Low & 4.67 & 1,006 & \multirow{2}{*}{$F(I, 42)=32.10, p<.001, \eta_{p}^{2}=.43$} & \multirow[t]{2}{*}{$F(I, 42)=107.47, p<.001, \eta_{p}^{2}=.72$} \\
\hline High & 13.80 & $\mathrm{I}, 264$ & & \\
\hline \multicolumn{5}{|l|}{ Group } \\
\hline Young adults & 11.29 & 1,232 & \multirow{2}{*}{$F(I, 42)=2.7 I, p=.107, \eta_{p}^{2}=.06$} & \multirow[t]{2}{*}{$F(I, 42)=11.78, p=.001, \eta_{p}^{2}=.22$} \\
\hline Older adults & 7.22 & $\mathrm{I}, 037$ & & \\
\hline \multicolumn{5}{|l|}{$\begin{array}{l}\text { Proactive interference } \times \\
\text { problem size }\end{array}$} \\
\hline $\begin{array}{l}\text { Proactive interference } \\
\text { effect (high minus low) in } \\
\text { small problems }\end{array}$ & 2.74 & 168 & \multirow[t]{3}{*}{$F(I, 42)=\mid 8.69, p<.00 I, \eta_{p}^{2}=.31$} & \multirow[t]{2}{*}{$F(I, 42)=17.16, p<.00 I, \eta_{p}^{2}=.29$} \\
\hline $\begin{array}{l}\text { Proactive interference } \\
\text { effect (high minus low) in } \\
\text { large problems }\end{array}$ & 15.52 & 348 & & \\
\hline \multicolumn{4}{|l|}{ Problem size $\times$ group } & \\
\hline $\begin{array}{l}\text { Problem size (large minus } \\
\text { small) effect in young adults }\end{array}$ & 17.10 & 355 & \multirow[t]{3}{*}{$F(I, 42)=4.61, p=.038, \eta_{p}^{2}=.10$} & \multirow[t]{2}{*}{$F(I, 42)=10.71, p=.002, \eta_{p}^{2}=.20$} \\
\hline $\begin{array}{l}\text { Problem size (large minus } \\
\text { small) effect in older adults }\end{array}$ & 9.77 & 173 & & \\
\hline \multicolumn{4}{|l|}{ Proactive interference $\times$ group } & \\
\hline $\begin{array}{l}\text { Proactive interference } \\
\text { effect (high minus low) in } \\
\text { young adults }\end{array}$ & 10.99 & 340 & \multirow[t]{2}{*}{$F(I, 42)=1.33, p=.255, \eta_{p}^{2}=.03$} & \multirow[t]{2}{*}{$F(I, 42)=10.83, p=.002, \eta_{p}^{2}=.21$} \\
\hline $\begin{array}{l}\text { Proactive interference } \\
\text { effect (high minus low) in } \\
\text { older adults }\end{array}$ & 7.27 & 176 & & \\
\hline
\end{tabular}

ANOVA: analyses of variance.

$18.69, p<.001, \eta_{\mathrm{p}}^{2}=.31$, showing that the proactive interference effect was larger in large problems (mean difference $[M D]=15.52 \%)$ than in small problems $(M D=$ $2.74 \%$ ). The main effect of group did not reach significance level, $F(1,42)=2.71, p=.107, \eta_{\mathrm{p}}^{2}=.06$. The interaction between problem size and group was significant, $F(1,42)=4.61, p=.038, \eta_{\mathrm{p}}^{2}=.10$. The problem size effect was significant in both groups $(p<.001)$ but was larger in young adults $(M D=17.10 \%)$ compared with older adults $(M D=9.77 \%)$. No interaction between proactive interference and group was observed, $F(1,42)=1.33$, $p=.255, \eta_{\mathrm{p}}^{2}=.03$. The three-way interaction between proactive interference, problem size, and group was not significant $(p=.422)$.

The same analysis on the median RTs of correct responses revealed a main effect of problem size, $F(1,42)$ $=89.72, p<.001, \eta_{\mathrm{p}}^{2}=.68$. Small problems were more rapidly solved than large problems (small problems: mean of median RTs $=1,003 \mathrm{~ms}, S E=23.05 \mathrm{~ms}$; large problems: mean of median RTs $=1,266, S E=38.49 \mathrm{~ms}$ ). The main effect of proactive interference was also significant, $F(1,42)=107.47, p<.001, \eta_{\mathrm{p}}^{2}=.72$. Low interfering problems (mean of median RTs $=1,006 \mathrm{~ms}, S E$ $=25.77 \mathrm{~ms}$ ) were more rapidly solved than high interfering ones (mean of median RTs $=1,264 \mathrm{~ms}, S E=35.64$ $\mathrm{ms}$ ). The interaction between problem size and proactive interference was found, $F(1,42)=17.16, p<.001$, $\eta_{\mathrm{p}}^{2}=.29$, showing that the proactive interference effect was greater in large problems $(M D=348 \mathrm{~ms})$ than in small problems $(M D=168 \mathrm{~ms})$. There was also a significant effect of group, $F(1,42)=11.78, p=.001, \eta_{\mathrm{p}}^{2}=.22$ , indicating that young adults were slower than older adults in multiplication solving. The interaction between problem size and group was observed, $F(1,42)=10.71, p$ $=.002, \eta_{\mathrm{p}}^{2}=.20$, (see Figure $\left.2 \mathrm{a}\right)$. The problem size effect was significant in both groups $(p<.001)$, but the mean difference between small and large problems was larger in young adults $(M D=355 \mathrm{~ms})$ than in older adults $(M D=$ $173 \mathrm{~ms})$. The proactive interference effect significantly interacted with the factor group, $F(1,42)=10.83, p=$ $.002, \eta_{\mathrm{p}}^{2}=.21$, (see Figure $2 \mathrm{~b}$ ). The proactive interference effect was significant in both groups $(p<.001)$ but was larger in young adults $(M D=340 \mathrm{~ms})$ than in older adults $(M D=176 \mathrm{~ms})$. The three-way interaction between 


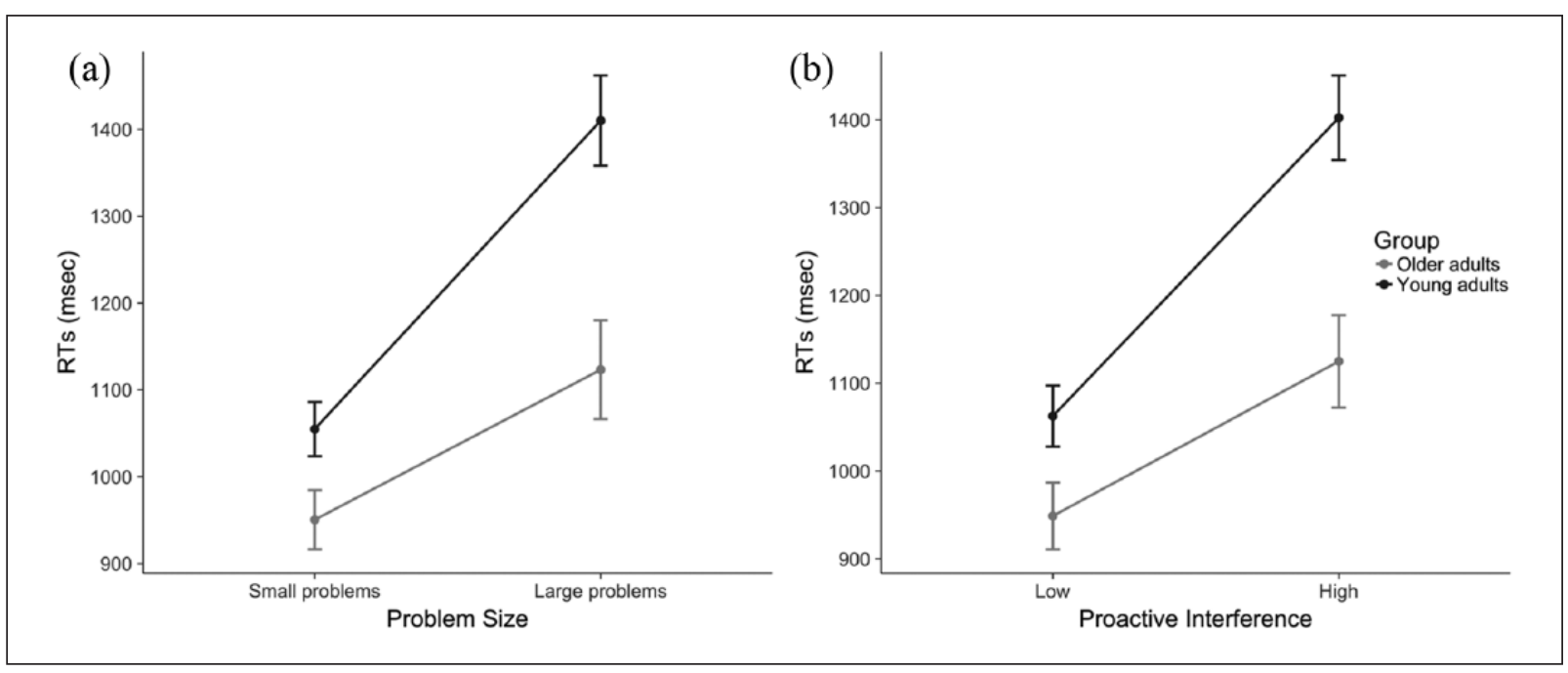

Figure 2. Mean of median RTs for (a) small and large problem sizes and for (b) low and high interference problems in young and older adults.

Table 2. Multiple regressions on the mean of median RTs with the interference parameter and the problem size as predictors in young and older adults.

\begin{tabular}{|c|c|c|c|c|c|c|c|}
\hline Reaction time & & $\begin{array}{l}\text { Zero-order } \\
\text { correlation }\end{array}$ & $\begin{array}{l}\text { Partial } \\
\text { correlation }\end{array}$ & $t$ & $p$ value & $\beta$ & $R^{2}$ \\
\hline \multirow[t]{2}{*}{$\begin{array}{l}\text { Young adults } \\
(N=24)\end{array}$} & $\begin{array}{l}\text { Interference } \\
\text { parameter }\end{array}$ & .70 & .53 & 3.60 & $<.005$ & .46 & .62 \\
\hline & Problem size & .69 & .51 & 3.39 & $<.005$ & .43 & \\
\hline \multirow[t]{2}{*}{$\begin{array}{l}\text { Older adults } \\
(N=20)\end{array}$} & $\begin{array}{l}\text { Interference } \\
\text { parameter }\end{array}$ & .69 & .54 & 3.66 & $<.005$ & .51 & .55 \\
\hline & Problem size & .61 & .38 & 2.40 & $<.050$ & .33 & \\
\hline
\end{tabular}

proactive interference, problem size, and group did not reach significance $(p=.560)$.

In a next step, we reanalysed the data using a regression analysis to explore which factors (problem size and/or proactive interference parameter) determine the difficulty across multiplications. Multiple regressions with median of RTs across problems as dependent variable and the proactive interference parameter and problem size as predictors were run for both groups (see Table 2). In young adults, the problem size as well as the proactive interference parameter were significant and showed high partial correlations and effect sizes. In older adults, the proactive interference parameter was significant and explained a substantial part of the variance in performance with a partial correlation of .54. The problem size factor was also significant. In line with the ANOVA analysis, both the problem size effect and the proactive interference effect substantially determined the performance in multiplication solving.

Finally, we performed separate regression analyses for each person with the RTs as the dependent variable and with the problem size and proactive interference parameter as predictors. The individual slopes for each independent variable were then used to examine group differences in sensitivity to problem size and to proactive interference. In agreement with the ANOVA analysis, independent samples $t$-tests revealed that older adults were less sensitive to proactive interference than young adults, $t(42)=-2.68$, $p=.010$. In contrast with the ANOVA, independent samples $t$-tests revealed that older adults were equally sensitive to problem size than young adults, $t(42)=-1.36, p=$ .181. To confirm that this null result denotes an absence of age-related difference in peripheral processes (null hypothesis), we performed a Bayesian independent samples t-test. $\mathrm{A} B F_{10}$ of .624 was observed providing anecdotal evidence for similar problem size effect between both groups. When the peripheral processes and the proactive interference are taken into account, the problem size effect remains constant with age. Given the partial overlap between both effects, the results obtained in the ANOVA for the problem size effect are probably driven by the proactive interference effect.

Regarding the peripheral processes, we compared the individual intercepts in order to investigate group differences. Surprisingly, independent samples $t$-tests showed no significant group effect in peripheral processes, $t(42)=0.19$, 
Table 3. The average error rate (in percentage; with standard error) and the mean of median RTs (in milliseconds; with standard error) by condition in young and older adults for the recent probes task.

\begin{tabular}{|c|c|c|c|c|c|c|c|c|c|c|c|c|}
\hline \multirow[t]{3}{*}{ Group } & \multicolumn{6}{|c|}{ Error rate (\%) } & \multicolumn{6}{|c|}{ Reaction times (ms) } \\
\hline & \multicolumn{2}{|c|}{ Positive } & \multicolumn{2}{|c|}{$\begin{array}{l}\text { Non-recent } \\
\text { negative }\end{array}$} & \multicolumn{2}{|c|}{$\begin{array}{l}\text { Recent } \\
\text { negative }\end{array}$} & \multicolumn{2}{|l|}{ Positive } & \multicolumn{2}{|c|}{$\begin{array}{l}\text { Non-recent } \\
\text { negative }\end{array}$} & \multicolumn{2}{|c|}{ Recent negative } \\
\hline & M & $S E$ & M & SE & M & $S E$ & $M$ & SE & $M$ & $S E$ & $M$ & $S E$ \\
\hline Young adults & 8.75 & 1.83 & 1.87 & 1.25 & 5.00 & 2.20 & 672.25 & 29.45 & 663.33 & 28.68 & 697.17 & 26.87 \\
\hline Older adults & 10.50 & 2.01 & 5.50 & 1.37 & 10.25 & 2.41 & 842.55 & 32.27 & 838.70 & 31.42 & 890.85 & 29.43 \\
\hline
\end{tabular}

M: mean; SE: standard error.

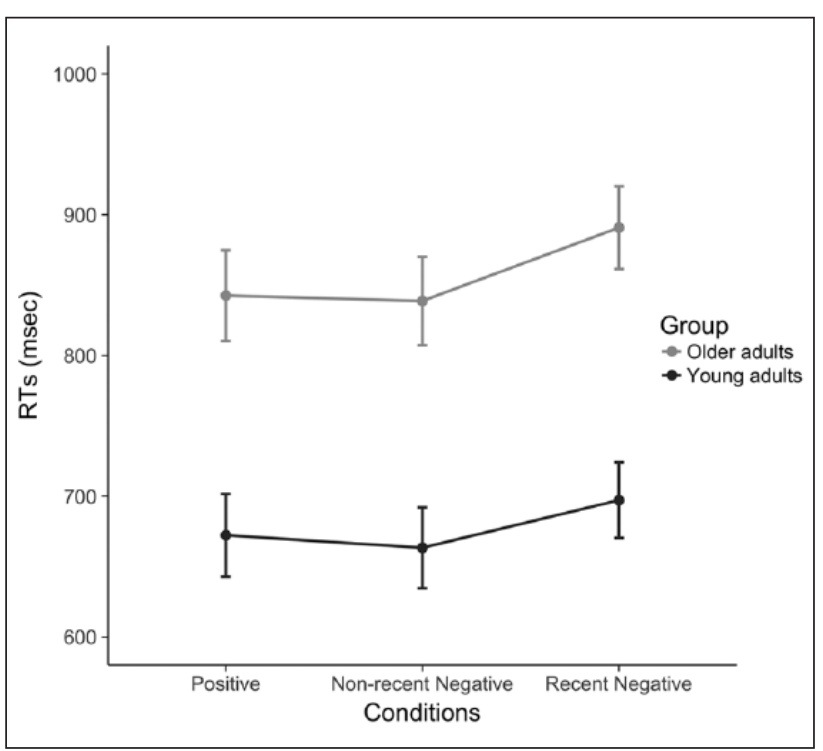

Figure 3. Mean of median reaction times for positive, nonrecent negative, and recent negative conditions in young and older adults.

$p=.849$. A Bayesian independent samples t-test. A $B F_{10}$ of .303 was observed providing substantial evidence for the null hypothesis. These results support similar peripheral processes between both groups.

In summary, we found no evidence for a decay in arithmetical facts with ageing. On the contrary, compared with young participants, older adults were faster, more accurate, less sensitive to the level of proactive interference, and equally sensitive to the problem size when solving single-digit multiplications.

\section{Recent probes task}

A repeated-measures ANOVA with condition (Positive, recent negative, non-recent negative) as within-subjects factor and group as between-subjects factor was performed separately on accuracy (percentage of errors) and on median RTs of correct trials. The average error rate and the mean of median RTs by condition for each group are provided in Table 3. Overall, error rate was on average $8.75 \%(S E=1.72 \%)$ and $5.21 \%(S E=1.57 \%)$ for older and young adults, respectively.

Analysis of errors revealed a main effect of condition, $F(2,84)=14.00, p<.001, \eta_{\mathrm{p}}^{2}=.25$. Post hoc comparisons with Bonferroni correction showed that more errors were produced in the recent negative condition compared with the non-recent negative condition $(p=.004)$ indicating a proactive interference effect (recent negative condition: error rate $=7.62 \%, S E=1.63 \%$; non-recent negative condition: error rate $=3.69 \%, S E=0.93 \%$ ). The main effect of group was not significant, $F(1,42)=2.31, p=.136$, $\eta_{\mathrm{p}}^{2}=.05$. The interaction between condition and group was not significant either, $F(2,84)=1.18, p=.313$, $\eta_{\mathrm{p}}^{2}=.03$. Although the interaction between condition and group was not significant, we decided to perform a planned comparison to directly compare the performance in the non-recent negative condition with the performance in the recent negative condition. This more direct measure of the difference in proactive interference effect between both age groups was not significant, $F(1,42)=0.51, p=.478$, $\eta_{\mathrm{p}}^{2}=.01$. Note that error rates were overall relatively low, making it possible that ceiling performance obscured agerelated differences in performance.

Analysis of RTs showed that the main effect of condition was significant, $F(2,84)=6.04, p=.004, \eta_{\mathrm{p}}^{2}=.13$. Post hoc comparisons with Bonferroni correction revealed that recent negative trials were responded slower than nonrecent negative trials $(p<.001)$, indicating a significant proactive interference effect (recent negative condition: mean of median RTs $=794 \mathrm{~ms}, S E=19.92 \mathrm{~ms}$; non-recent negative condition: mean of median RTs $=751 \mathrm{~ms}, S E=$ $21.27 \mathrm{~ms})$. The main effect of group was also significant, $F(1,42)=21.11, p<.001, \eta_{\mathrm{p}}^{2}=.33$. Older adults (mean of median RTs $=857 \mathrm{~ms}, S E=28.90 \mathrm{~ms}$ ) were globally slower than younger adults (mean of median RTs $=678$ $\mathrm{ms}, S E=26.38 \mathrm{~ms}$ ). The interaction between condition and group failed to reach significance, $F(2,84)=0.42, p=.655$, $\eta_{\mathrm{p}}^{2}=.01$, (see Figure 3 ). The Bayes factor model comparison yielded evidence for an absence of interaction between condition and group. The best model was the additive model (with main effects of both condition and 


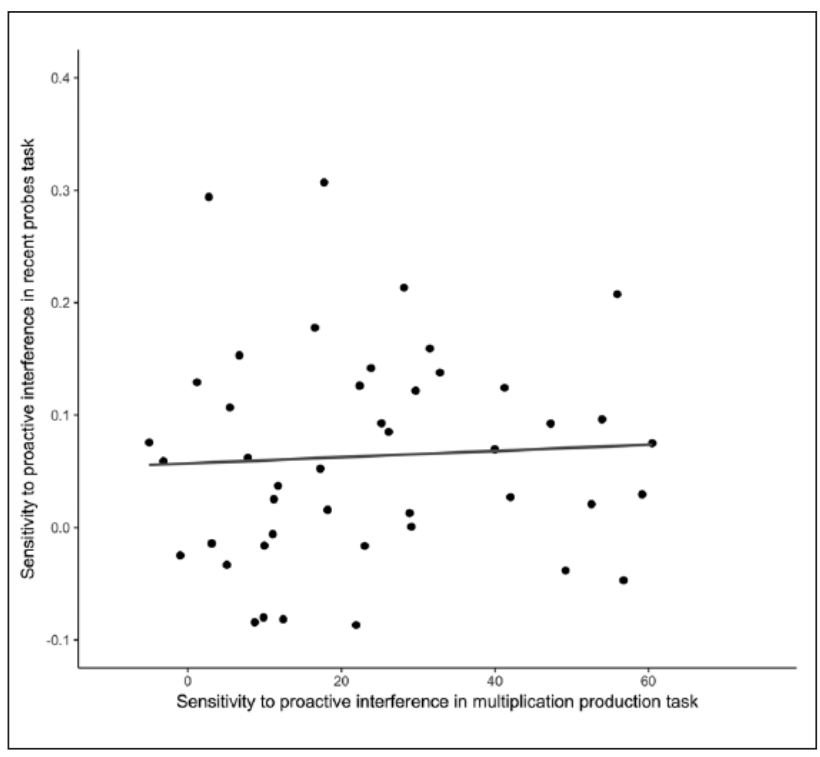

Figure 4. The absence of correlation between the sensitivity to proactive interference in the numerical and non-numerical domains. Scatter plot of the correlation between the sensitivity to proactive interference in multiplication production task (X-axis) and the sensitivity to proactive interference in recent probes task ( $\mathrm{Y}$-axis).

group but with no effect of interaction condition $\times$ group). This model was preferred by a factor of 6.192 over the full model (with both main effects and an interaction).

We further evaluated the age effect in the sensitivity to interference by directly comparing the individual measures of proactive interference between the two groups. Confirming the results of the repeated-measures ANOVA, independent samples $t$-tests showed no significant group effect in sensitivity to proactive interference, $t[42]=0.45$, $p=.655$. We performed a Bayesian independent samples t-test to confirm the null hypothesis. A $B F_{10}$ of .326 was observed providing substantial evidence for the null hypothesis. This supports that the sensitivity to proactive interference did not differ between young and older adults.

\section{Relation between the sensitivity to proactive interference in numerical and non-numerical domain}

Pearson's correlation between the individual sensitivity to proactive interference in multiplication solving and in recent probes task was carried out for all subjects. No correlation was found, $r(44)=.05, p=.724$, (see Figure 4). A $B F_{10}$ of .200 was observed for this correlation, indicating substantial evidence in favour of the null hypothesis.

\section{Discussion}

The main aim of this study was to investigate possible agerelated changes of the problem size effect and the proactive interference effect in AF solving. To this end, the performance of young and older adults was compared in a multiplication production task. Furthermore, an independent measure of proactive interference was assessed using a recent probes task with letters to further define the architecture underlying the proactive interference effect in multiplication solving.

In the multiplication production task, the results including both an ANOVA and regression analyses showed that the performance in AF solving was influenced by both the problem size effect and the proactive interference effect in older adults. Replicating previous findings (De Visscher \& Noël, 2014), the same pattern of results is observed in young adults. The problem size effect was robustly observed in young and older adults, meaning that in the two groups, RTs and error rates increased as the size of the problem increased. Furthermore, the problem size effect observed in older adults was similar as the one observed in younger adults when peripheral processes and proactive interference were taken into account. In sum, retrieval of AFs from long-term memory was not affected by ageing. This conclusion is consistent with previous studies observing equal effect sizes between young and older adults (Allen et al., 2005, 1997; Geary et al., 1993; Geary \& Wiley, 1991).

Regarding the proactive interference effect, to our knowledge, no study has yet examined it in the context of multiplication solving in older adults. The results showed that the proactive interference parameter substantially determined the performance across multiplication problems not only in young adults, thus replicating previous results described in the literature (De Visscher et al., 2015; De Visscher \& Noël, 2014), but also in older adults, with greater interference leading to longer RTs and to larger error rate. Moreover, the comparison of sensitivity to proactive interference between both groups revealed that older adults were less sensitive to interference than younger adults. The proactive interference parameter represents the proactive interference weight for each multiplication based on the physical similarity of digits between a new problem to store and the previously learned ones. This proactive interference effect is believed to be decisive at the encoding stage, when children were learning multiplication tables (De Visscher \& Noël, 2014). Corroborating this assumption, our results indicate that the proactive interference effect continues to impact multiplication performance into adulthood but progressively decreases its impact with age, probably due to the growing gap with the moment of $\mathrm{AF}$ acquisition: because $\mathrm{AF}$ network of older adults is established since a long time, they have overcome a part of the proactive interference effect. Besides this developmental explanation, the weaker sensitivity to proactive interference observed in older adults could also be related to differences in strategy selection. That is, due to differences in educational practices, older adults may have used the retrieval strategy more frequently/efficiently than 
younger adults. Note, however, that such explanation hold can only be validated if future studies can demonstrate that a relation exists between strategy use and the proactive interference parameter.

In the recent probes task with letters, results indicated that both young and older adults were sensitive to proactive interference. Contrary to earlier observations (e.g., Jonides et al., 2000), this effect did not differ between older and young adults. Furthermore, no relation was found between the sensitivity to proactive interference in the letter task and in the multiplication task. A possible explanation for the absence of this relation is that the origin of proactive interference is different in both tasks. Indeed, the proactive interference in the multiplication task arises from the storage of $\mathrm{AFs}$ and, as a consequence, from long-term memory. Conversely, proactive interference comes from short-term memory in the recent probes task.

However, recent studies found such relation in a single case study of a person suffering from specific and developmental AF retrieval deficits as well as in the comparison of children with good or weak AF network (De Visscher \& Noël, 2013, 2014). Taken together with our results, it seems that this relation exists in developmentally young or in clinical populations but is not observed in non-clinical adult populations. Thus, the general capacity of resistance to proactive interference (with non-numerical material) would play a key role during the development of the AF network. To explain this pattern of results, we can use the analogy of constructing a building. The construction of a building requires the use of scaffoldings to support the walls. The use of scaffoldings will only be necessary during the process of construction, and therefore, its implication in the construction will be visible at this moment only. Once built, the scaffoldings are no longer necessary, and its relation with the building will no longer be visible (for further details on this analogy, see Apperly, Samson, \& Humphreys, 2009). Similarly, the general capacity of resistance to proactive interference seems to be involved in the development of the AF network, explaining the effect of the proactive interference parameter. Once AFs are acquired, we continue to observe the specific effect of proactive interference in multiplication solving with the most interfering problems leading to longer RTs and to larger error rate. However, according to this view and in line with our results, the proactive interference in AF solving in adulthood should not be influenced by changes in the general capacity of resistance to proactive interference. Nevertheless, one could hypothesise that if we were teaching these participants new arithmetical facts, then the influence of general capacity of resistance to proactive interference would become apparent again. However, even in those conditions, group differences might not be expected as the older participants did not show larger sensitivity to proactive interference than younger participants in the recent probe task.
In conclusion, this study shows that when solving multiplications, both young and older adults were sensitive to the effects of interference and of the problem size. That is, both interference and problem size affected performance negatively: the time needed to solve a multiplication problem increases as the level of interference and the size of the problem increase. Consistent with previous research (e.g., Allen et al., 2005), the problem size effect remains constant with age, indicating a preserved AF network in older adults. Interestingly, we also observed that the sensitivity to proactive interference in multiplication solving was weaker in older than young adults. Finally, our results suggest that the general capacity of resistance to proactive interference in adulthood is no longer involved in the proactive interference effect in numerical domain.

\section{Declaration of conflicting interests}

The author(s) declared no potential conflicts of interest with respect to the research, authorship, and/or publication of this article.

\section{Funding}

The authors disclosed receipt of the following financial support for the research, authorship, and/or publication of this article: Kim Archambeau is supported by the Belgian Fonds National de la recherche Scientifique (FNRS) when the study was conducted (grant number FC 4247).

\section{Supplementary material}

The supplementary material is available at: qjep.sagepub.com

\section{Note}

1. Using the recent negative condition as the baseline yielded the same pattern of results.

\section{References}

Allen, P. A., Bucur, B., Lemaire, P., Duverne, S., Ogrocki, P. K., \& Sanders, R. E. (2005). Influence of probable Alzheimer's disease on multiplication verification and production abstract. Aging, Neuropsychology, and Cognition, 12(1), $1-31$.

Allen, P. A., Smith, A. F., Jerge, K. A., \& Vires-Collins, H. (1997). Age differences in mental multiplication: Evidence for peripheral but not central decrements. The Journals of Gerontology: Series B, 52, 81-90.

Apperly, I. A., Samson, D., \& Humphreys, G. W. (2009). Studies of adults can inform accounts of theory of mind development. Developmental Psychology, 45, 190-201.

Ashcraft, M. H. (1982). The development of mental arithmetic: A chronometric approach. Developmental Review, 2, 213236.

Ashcraft, M. H. (1987). Children's knowledge of simple arithmetic: A developmental model and simulation. In J. Bisanz, C. J. Brainerd \& R. Kail (Eds.), Formal methods in developmental psychology (pp. 302-338). New York, NY: Springer.

Ashcraft, M. H. (1992). Cognitive arithmetic: A review of data and theory. Cognition, 44, 75-106. 
Ashcraft, M. H., \& Christy, K. S. (1995). The frequency of arithmetic facts in elementary texts: Addition and multiplication in grades 1-6. Journal for Research in Mathematics Education, 26, 396-421.

Ashcraft, M. H., \& Guillaume, M. M. (2009). Chapter 4: Mathematical cognition and the problem size effect. In B. Ross (Series Ed.), The psychology of Learning and Motivation (Vol. 51, pp. 121-151). Cambridge, MA: Academic Press.

Boles, D. B., \& Clifford, J. E. (1989). An upper- and lowercase alphabetic similarity matrix, with derived generation similarity values. Behavior Research Methods, Instruments, \& Computers, 21, 579-586.

Campbell, J. I. (1995). Mechanisms of simple addition and multiplication: A modified network-interference theory and simulation. Mathematical Cognition, 1, 121-164.

Campbell, J. I., \& Graham, D. J. (1985). Mental multiplication skill: Structure, process, and acquisition. Canadian Journal of Psychology/revue Canadienne De Psychologie, 39, 338-366.

Campbell, J. I., \& Xue, Q. (2001). Cognitive arithmetic across cultures. Journal of Experimental Psychology: General, 130, 299-315.

Cerella, J. (1991). Age effects may be global, not local: Comment on Fisk and Rogers (1991). Journal of Experimental Psychology: General, 120, 215-223.

Chiappe, P., Siegel, L. S., \& Hasher, L. (2000). Working memory, inhibitory control, and reading disability. Memory \& Cognition, 28, 8-17.

De Brauwer, J., Verguts, T., \& Fias, W. (2006). The representation of multiplication facts: Developmental changes in the problem size, five, and tie effects. Journal of Experimental Child Psychology, 94, 43-56.

De Visscher, A., Berens, S. C., Keidel, J. L., Noël, M.-P., \& Bird, C. M. (2015). The interference effect in arithmetic fact solving: An fMRI study. NeuroImage, 116, 92-101.

De Visscher, A., \& Noël, M.-P. (2013). A case study of arithmetic facts dyscalculia caused by a hypersensitivity-to-interference in memory. Cortex, 49, 50-70.

De Visscher, A., \& Noël, M.-P. (2014). The detrimental effect of interference in multiplication facts storing: Typical development and individual differences. Journal of Experimental Psychology: General, 143, 2380-2400.

Eidswick, J. (2012). A Bayesian alternative to null hypothesis significance testing. Shinken Research Bulletin, 16(1), 2-15.

Geary, D. C. (1993). Mathematical disabilities: Cognitive, neuropsychological, and genetic components. Psychological Bulletin, 114, 345-362.

Geary, D. C., Frensch, P. A., \& Wiley, J. G. (1993). Simple and complex mental subtraction: Strategy choice and speedof-processing differences in younger and older adults. Psychology and Aging, 8, 242-256.

Geary, D. C., \& Wiley, J. G. (1991). Cognitive addition: Strategy choice and speed-of-processing differences in young and elderly adults. Psychology and Aging, 6, 474-483.

Hamann, M. S., \& Ashcraft, M. H. (1986). Textbook presentations of the basic addition facts. Cognition and Instruction, 3, 173-202.

Imbo, I., \& Vandierendonck, A. (2008). Effects of problem size, operation, and working-memory span on simple-arithmetic strategies: Differences between children and adults? Psychological Research, 72, 331-346.
Jonides, J., Marshuetz, C., Smith, E. E., Reuter-Lorenz, P. A., Koeppe, R. A., \& Hartley, A. (2000). Age differences in behavior and PET activation reveal differences in interference resolution in verbal working memory. Journal of Cognitive Neuroscience, 12, 188-196.

Jordan, N. C., \& Montani, T. O. (1997). Cognitive arithmetic and problem solving a comparison of children with specific and general mathematics difficulties. Journal of Learning Disabilities, 30, 624-634.

LeFevre, J.-A., Bisanz, J., Daley, K. E., Buffone, L., Greenham, S. L., \& Sadesky, G. S. (1996). Multiple routes to solution of single-digit multiplication problems. Journal of Experimental Psychology: General, 125, 284-306.

Loosli, S. V., Rahm, B., Unterrainer, J. M., Weiller, C., \& Kaller, C. P. (2014). Developmental change in proactive interference across the life span: Evidence from two working memory tasks. Developmental Psychology, 50, 1060-1072.

Love, J., Selker, R., Marsman, M., Jamil, T., Dropmann, D., Verhagen, A. J., . . . Wagenmakers, E.-J. (2015). [Computer Software]. Journal of Applied Social Psychology.

May, C. P., Hasher, L., \& Kane, M. J. (1999). The role of interference in memory span. Memory \& Cognition, 27, 759-767.

McCloskey, M., \& Lindemann, A. M. (1992). Chapter 10: Mathnet: Preliminary results from a distributed model of arithmetic fact retrieval. In J. I. D. Campbell (Ed.), Advances in psychology (Vol. 91, pp. 365-409). Cambridge, MA: Academic Press.

Miller, K., Perlmutter, M., \& Keating, D. (1984). Cognitive arithmetic: Comparison of operations. Journal of Experimental Psychology: Learning, Memory, and Cognition, 10, 46-60.

Monsell, S. (1978). Recency, immediate recognition memory, and reaction time. Cognitive Psychology, 10, 465-501.

Nasreddine, Z. S., Phillips, N. A., Bédirian, V., Charbonneau, S., Whitehead, V., Collin, I., \& Chertkow, H. (2005). The Montreal Cognitive Assessment, MoCA: A brief screening tool for mild cognitive impairment. Journal of the American Geriatrics Society, 53, 695-699.

Penner-Wilger, M., Leth-Steensen, C., \& LeFevre, J.-A. (2002). Decomposing the problem-size effect: A comparison of response time distributions across cultures. Memory \& Cognition, 30, 1160-1167.

Stazyk, E. H., Ashcraft, M. H., \& Hamann, M. S. (1982). A network approach to mental multiplication. Journal of Experimental Psychology: Learning, Memory, and Cognition, 8, 320-335.

Thompson-Schill, S. L., Jonides, J., Marshuetz, C., Smith, E. E., D'Esposito, M., Kan, I. P., ... Swick, D. (2002). Effects of frontal lobe damage on interference effects in working memory. Cognitive, Affective, \& Behavioral Neuroscience, $2,109-120$.

Verguts, T., \& Fias, W. (2005). Interacting neighbors: A connectionist model of retrieval in single-digit multiplication. Memory \& Cognition, 33(1), 1-16.

Wagenmakers, E.-J., Wetzels, R., Borsboom, D., \& van der Maas, H. L. J. (2011). Why psychologists must change the way they analyze their data: The case of Psi: Comment on Bem (2011). Journal of Personality and social Psychology, 100, 426-432.

Zbrodoff, N. J., \& Logan, G. D. (2005). What everyone finds: The problem-size effect. In J. I. D. Campbell (Ed.), Handbook of mathematical cognition (pp. 331-345). New York, NY: Psychology Press. 